\title{
LÉGISLATION
}

\section{Un exemple de responsabilité civile d'un Concessionnaire à propos d'un arrêt de la Cour d'Alger du 30 décembre 1925}

\author{
Par Paul BOUgault, Avocat ò la Cour d'Appel de Lyon.
}

\begin{abstract}
La loi du 15 Juin 1906 sur les distributions d'énergie ne contient pas seulement des dispositions d'ordre lechnique, administratif ou contractuel; elle renferme divers articles qui menacent d'instances correctionnelles les distribuleurs qui ne seraient pas en règle avec les prescriptions imposées dans l'intérèl de la sécurité des personnes. Bien que l'arrêt que nous allons reproduire ci-dessous n'émane pas d'une Juridiction répressive, il s'est inspiré au point de vue simplement de la question de la compétence, en matière d'accident, de certains principes qu'il est utile de connatire.
\end{abstract}

Exposé des faits qui ont donné lieu au procès. -- Dans la nuit du 19 au 20 aô̂t 1924, les époux Casanova demeurant à Saint-Eugène (Algérie) etaient dans leur chambre, quand ils furent gravement brûlés par des décharges électriques provenant des fils d'éclairage situés dans leur maison : ces fils avaient momentanément rencontré ceux d'un transport de force, par suite de la chute d'un poteau, renversé sur la route par le chauffeur de la Compannie Anonyme de Transports Automobiles, conme sous le nom de SATA. Les époux Casanova assignèrent devant le tribunal Civil d'Alger la Compagnie distributrice (Compagnie Centrale d'éclairage par le Gaz Lebon et Cie) pour obtenir une forte indemnite.

Cette Compagnie souleva l'incompétence du Tribunal judiciaire, en arguant que sa ligne était destince à distribuer la lumière et la force motrice dans la commune de St-Eugène, pour l'usage public; que son installation avait été permise par arrêté du Préfet d'Alger du 14 janvier 1924; que les plans et épures en ont été établis conformément à la réglementation légale et approuvés, avec autorisation de construire, par le service compétent; que l'on pouvait donc trouver dans son occupation du sol public tous les éléments caractéristiques d'un travail public.

Elle demandait donc à être jugée par les Tribunaux administratifs et insistail sur les principes admis par la Jurisprudence qui considere la Juridiction administrative comme le Tribunal normal, à l'égard des accidents causés par les concessionnaires ou les entrepreneurs d'un travail public.

Le Tribunal d'Alger et, sur appel, la Cour d'Alger (cetle dernière par un arrèt du 30 décembre 1925 reproduit ci-dessons et rapporté dans le Recueil hebdomadaire de Dalloz 1925, page 154) ont admis la compétenee du Tribumal judiciaire pour les trois nolifs suivanls :

$1^{0}$ Si le concessionnaire est, en principe, justiciable du Conseil de Préfechure quand il cause un accident, la Juridiction du Tribunal civil reprend tout son empire, quand le fait dommageable allégué par la victime, présente le caractère d'un délit ou d'une contravention.

$2^{\circ} \mathrm{Or}$, on retrouvait, dans l'espèce, des faits qui auraient pu rendre applicable l'article 25 de la loi du 15 juin 1906, c'est-à-dire rendre compétente la Juridiction régressive en raison d'infractions commises dans l'intérêt de la sécurité des personnes.

$3^{\circ}$ Enfin, les époux Casanova avaient passé avec la Compagnie distributrice un contrat connu sous le nom de "police "qui devait leur assurer, dans leur domicile, la réception d'un courant non dangereux, et c'est en vertu de ce contrat que le fil, générateur de l'accident, avait été placé dans leur demeure.

Objet de la présente étude. - Nous n'avons point à parler du troisième motif qui peut ètre allégué par toutes les personnes qui ont une police d'éclairage et sont victimes chez elles d'un accident causé par les fils d'adduction qui pénètrent dans leur immeuble (voir sur ce point les arrêts célèbres de la Courd'Appel de Grenoble du 22 février 1913, affaire Drevelon et Serpollet analysés dans mon étude parue en septembre 1914, aux communications du contentieux de la Chambre Syndicale des Forces hydrauliques no 256 , page 409 ).

Mais, les deux premiers ont une importance considérable, car ils régissent la matière des accidents occasionnés sur la voie publique et leur application est autrement large.

Nous examinerons les principes, les précédents et les textes sur lesquels la Cour d'Appel d'Alger a pu s'appuyer pour dire qu'un concessionnaire (de distribution d'énergie ou tout autre) ne peut réclamer son renvoi devant la Juridiction administrative quand le fait accidentel provient d'une circonstance assimilable à un délit ou à une contravention, et pour admettre que dans le cas particulier, elle decouvrait une circonstance de cette nature.

Il nous paraît également, utile puisque nous étudions une affaire de nature exceptionnelle, de jeter ensuite un regard sur le cas général, afin d'éviter toute équivoque dans l'avenir : quel est le Tribunal compétent pour statuer sur un accident normal, imputable à un concessionnaire.

Enfin, par un enchaînement tout naturel, nous serons conduits a nous demander si l'exception que nous commençons par relater, est la seule qui puisse se produire, ou bien s'il en existe une autre. Cette matière si importante de la compétence en ce qui concerne les accidents, sera ainsi complètement examiné. 
Accident imputable à une circonstance délictuelle. - Ia Cour d'Alger a statué en ces termes : "Attendu que les Tribunaux "Civils sont compélents pour prononcer sur la responsabilité " du concessionnaire de travaux publics résultant des dom" mages causés aux particuliers, lorsque le fait dommageable " allégué présente le caractère d'un délit ou d'une contravention ; "que ledit délit ait été on non poursuivi devant la Juridiction "répressive. "Cette affirmation de la Cour d'Appel d'Alger est parfaitement conforme à la Jurisprudence, au moins actuelle, de la Cour do Cassation. Dans son livre récent (1927) "Traité elémentaire du contentieux administratif " M. Jean Applelon s'exprime ainsi : (voir no231, pages 424 el suivantes) "La Cour " de Cassation, jusqu’à ces dernières années, après avoir paru " adhérer à la Jurisprudence du Conseil d'Etat, et du Tribunal " des Conflits, a fait de grands cfforts pour en restreindre lappli" cation. Elle y est arrivée par deux procédés : toul d'abord, " elle entend réserver aux Tribumaux Judiciaires le jugement "de toute demande en indemmité fondée sur un accident à "l'origine duquel on peut relever un délit pénal, généralement "le délit d'homicide on de blessure par imprudence. L'autorité " Judiciaire, d'après elle, est compétente pour comnaître de " ces demandes, que laction civile soit exercée ou non concur"remment avec l'action publique, q'uelle soit dirigée contre " l'auteur du délit, ou contre la personne civilement responsable "(Ch. Civ. 27 novembre 1918, D. P. 1919. I. 17 ; \& janvier 1923, " D.P. 1923. I. 33)." .

Il est certain que l'arrèt cité par M. Appleton celui du 27 novembre 1918, connu sous le nom de l'arrêt Somerville contre Compagnie des Tramways de Nice (1) est extrêmement précis : dans cette affaire, il s'agissait d'un accident survenu à une persomne par le fait d'un wagon dévalant rapidement d'une voie en construction; l'arrêt attaqué avait constaté que l'imprudence des employés de la Compagnie qui avait laissé, sans l'avoir calé, un wagon au sommet d'une pente, était punissable d'après les articles 319 et 320 du Code Pénal : et la Cour ajoute : attendu " que si, en vertu de la loi du 28 Pluviose an 8, art. 4 alinéa 3, "l'autorité administrative est scule compétente pour prononcer " sur la responsabilité résultant des torts ct dommages causés " aux particuliers, par le fait personnel des entrepreneurs, " lorsque ce fait se rattache directement à l'exécution ou l'inexé" cution d'un travail public, il en est autrement lorsque le fait " allégué présente le caractère d'un crime d'un délit ou d'une " contravention." (2) Cette Jurisprudence affirmée dans un arrêt récent, mais qui peut se recommander de quelques précé-

\section{(1) L'arrêt du 8 janvier 1923 sera examiné plus loin.}

(2) Dans une note très substantielle parue dans Dalloz 1919. I. page 17 , sous cet arrêt, M. Appleton fait remarquer que cette Jurisprudence de la Cour Suprême, est basée sur l'article 1382 du Code Civil ; même lorsque l'Administration est en cause. La Cour de Cassation tend à admettre que la réparation est due parce qu'il a été commis une faute, et le comble de la faute n'est-il pas dans un " délit " commis ? Au contraire, le Conseil d'Etat fait dériver d'une question d'équité le principe de la réparation. Il veut que les charges des services publics soient égales pour tous, et que les inégalités graves et personnelles soient compensées par rles indemnités pécuniaires. I,e Conseil d'Etat tire de là cette conséquence précieuse pour les victimes d'accidents, que cette action n'est influencée en rien par l'existence et l'exercice de l'action publique (Cons. Et., 22 mai 1912, D. P. 1915. 3. 3, affaire Augé : dans cette affaire le Conseil d'Etat a dit que la mère de la victime d'un accident survenu pendant l'exécution d'un travail public, au licu d'intenter une action civile née d'un clélit dé homicide pour imprurdence, a intenté devant le Conseil de Préfecture une action en réparation d'un dommage causé par l'exécution d'un travail public, la prescription qui aurait été applicable à la première, ne l'est pas à la secondc.) dents, obligeail la Cour à rechercher immédiatement si, dans l'espèce, on ne trouvait pas au moins à l'élat latent, un délit qui aurail pu ètre relevé (voir notamment 24 mai 1886, D). P. 87. 1. 7., alfaire Compagnie Générale des Tramways contre Roussel el Carbond el divers arréts cilés dans la note de M. $\Lambda$ ppleton, D. P. 1923. I. 33).

Faits considérés comme délictuels. - I a Cour d'Alger a relenu comme delictuels les faits suivants :

a) Ia ligne aurait été mise e'll service avant qu'ait été délivrée l'autorisation prefectorale de circulation de courant prévue par l'article 15 de la loi da 15 juin 1906 ;

b) La mise sous tension de la ligne aurait eu lieu sans que toutes les précautions que commandait le rapprochement des conducteurs de haule el basse tension eussent élé prises.

Sans doute, lauleur responsable de l'accident était bien le conducteur de la voilure de la Compagnie anonyme des Transports automobiles, qui avait renversé le poteau; mais, celle responsabilité ne laisant pas disparaître celle de la Compagnie distributrice, en raison des deux manquements qui viemnent d'être indiqués.

Or, l'art. 15 de la loi du 15 juin 1906 est ainsi concu : La mise " en service d'une distribution d'énergie électrique, ne peut " avoir lieu qu’à la suite des essais faits en présence du service du " contrôle el des représentants des services intéressés, el après "délivance, par le Préfel. d'une aulorisation de circulation "de courant."

D'autre part, l'article 19 déclare que " des arrêtés pris par "le Ministre des Travaux Publics... apres avis du Comité d'Elec" tricité déterminent les conditions techniques auxquelles devront " satisfaire les distributions d'énergie au point de vue de la sécu"rité des persomnes el des services publics intéressés."

Enfin, l'article 25 de celte même loi déclare que "Loute infrac" Lion aux dispositions édictées dans l'intérèt de la sécurité des "personnes, soit par des règlements d'administration publique, " soit par les arrêtés visés à l'article 19, sera poursuivie devant "les Tribunaux correctionnels et pumis d'une amende.. etc. ".

L'action ne se prescrit que par trente ans; l'acquittement de l'auteur de la faute qui a pu entrainer l'accident, sera sans influence sur le résultat de l'action en dommages intérêts devant les Tribunaux administratifs, car la responsabilité de la puissance publique est indépendante de toute idée de faute (Hauriou, page 376, édition de 1921).

I.a Cour de Cassation, au contraire, présente une jurisprudence très dangereuse pour les victimes d'un accident qui auront à tenir compte, non seulement de la prescription de 3 ans, qui est libératoire pour l'auteur d'un délit, même au point de vue de la réparation civile, mais aussi de l'acquittement du prévenu qui empichera ladite réparation, si celle-ci est demandée en raison des faits identiques, sans élément nouveau.

M. Appleton constate aussi que s'il y a unanimité à reconnaître que l'administration n'est pas un "commettant "à l'égard de ses agents, on ne comprend plus pour quel motif la responsabilité de l'administration serail lice à l'application de l'art. 1381 du colle Civil.

Enfin, d'après lui, en 1919, le Tribunal des Conflits n'aurait pas encore eu à prendre une ligne de conduite bien tranchée, en matière de concession. Il constate toutefois qu'il a eu à admettre la compétence administrative lorsque l'action est dirigée contre l'Administration même dans le cas où il existait un délit imputable aux agents de celle-ci (Trib. des Conf., 8 nov. 1902. D. 1901. 3. 36 aff. Loquen). 
Il n'en fallait pas davantage pour que la Cour estimât qu'elle se trouvait en présence d'une série d'infractions ayant pour but de compromettre la sécurité des personnes ; car c'est notamment au point de vue de cette sécurité que la mise en service doit être retardée jusqu'à l'autorisation préfectorale de circulation de courant qui est donnée après les essais réglementaires : elle a donc tranché le différend en faveur de la compélence judiciaire : car bien qu'une poursuite ne fut pas intervemue, devant le Tribunal répressif, les faits allégués devant le Tribunal Civil était do ceux qui auraient pu la motiver; aussi la Cour a-l-elle statué ainsi : " allendu qu'une des causes de l'accident consiste dans " une infraction aux dispositions d'ordre pénal de l'article 15 " de la loi du 15 juin 1906 et aux dispositions de l'article 3201 du "Code Pénal.» (Ce dernier article est celui qui est commu par le public sous le nom de "répression des blessures par imprudence").

Sommaire et texte de l'arrêt. - L Les tribunaux civils sont compétents pour prononcer sur la responsabilité du concessionnaire de travaux publics résultant des dommages causés aux particuliers, lorsque le fait dommageable présente le caractère d'un délit on d'une contravention, que le délit ait élé on non poursuivi devant la juridiction répressive;

Une compagnie concessiomnaire d'éclairage électrique ayant commis la faute de mettre en service la ligne électrique sans avoir pris les précautions que le rapprochement des conducteurs de haute el de basse tension devait entrâner, a commis une infraction aux dispositions d'ordre pénal de l'art. 25 de la loi des 15 et 17 juin 1906, et aux dispositions de l'art. $320 \mathrm{c}$ pén. ;

En conséquence les demandes d'indemnité formées par les particuliers victimes d'un accident, conséquence de cette infraction, sont de la compétence des tribunaux judiciaires.

\section{I.A COUR ;}

ArR̂ेT

Attendu que les époux Casanova exposent dans leur assignation du 9 février 1925 que pendant la muit du 19 au 20 août 1921, ils étaient conchés lorsqu'ils ont été cruellement brâlés par des décharges électriques provenant des fils d'éclairage installés dans leur maison par la Compagnie Lebon, pour leur fournir la lumière électrique ; que cet accident a été causé par la rupture d'un poteau supportant une ligne électriçue d'éclairage et de force, dont la Compagnie Lebon est concessiomaire, qui a mis en contact les fils d'éclairage et les fils de force motrice; que ce poteau a été renversé par le sieur Baba-Aïsa Foudil ben Smaïl, chauffeur préposé de la Société Anonyme de Transports automobiles dite Compagnie S.A.T.A.;

Attendu que les époux Casanova ont assigné la Compagnie S.A.T.A. et la Compagnie Lebon devant le tribunal civil d'Alger pour s'entendre condamner a payer conjointement ot solidairement à titre de dommages-intérèts 400.000 franes au sieur Casanova et 100.000 francs a la dame Casanova ;

Attendu que la Compagnie Lebon a conch à l'incompétenee du tribunal civil d'Alger rutione muleria, la juridiclion administrative étant seule compétente pour statuer sur la demande; que le tribunal civil d'Alger s'est déclaré compétent :

Altendu que la Compagnie Vabon a régulioroment interjoti appel de cette décision; que les époux Casanova en demandent la confirmation; que la Socicté S.A.T.A. a fait siemnes les conclusions des époux Casanova sur la compétence;

Atlendu que les époux Casanova attribuent l'accident en co qui concerne la responsabilité de la Compagnic Lebon, à deux causes : $1^{\circ}$ les défectuosités de l'installation de la ligne électrique ; $2^{\circ}$ à l'inexécution de la police d'abonnement aux termes de laquelle ladite compagnie devait leur fournir l'éclairage dans des conditions exemptes de tout danger;

Atlendu que la Compagnie J.ebon fonde l'exception d'incompélence qu'elle a soulevée sur les dispositions de l'art. 4 de la loi du 28 pluv. an 8 , aux termes duquel il appartient aux seuls tribunaux administratifs de connaître des actions en réparation du dommage cue l'établissement ou l'utilisation d'un ouvrage ayant le caractire de travaux publics peuvent causer à des particuliers; qu'a l'appui de sa prétention elle articule que la ligne électrique sur laquelle s'est produit l'accident est destinée à distribuer la lumière et la force électrique dans la commune de Saint-Eugene pour l'usage public; que son installation a été permise par arrèté du préfet d'Alger du 11 janvier 1924; que les plans et épures en ont été établis conformément à la réglementation légale et approuvés avec l'autorisation de construire par le service administratif compétent le 10 février; qu'ainsi se trouvent réunis les éléments caractéristiques d'un service public;

Attendu qu'aux termes des lois des 15 et 17 juin 1906, la mise en service d'une distribution d'énergie électrique ne peut avoir lieu qu'à la suite des essais faits en présence du service du contrôle et du représentant du service intéressé et après délivrance par le prefet d'une autorisation de circulation (art. 15); que toute infraction aux dispositions édictées dans l'intérêt de la sécurité des personnes, soil par des règlements d'administration publique, soit par des arrètés visés à l'art. 19, doit être poursuivie devant les tribumaux correctionnels et punie d'une amende de 16 franes à 3.000 francs, sous préjudice des pénalités prévues par le code pénal, en cas d'accident résultant de l'infraction;

Attendu que d'un procès-verbal établi le 20 août 1924, par M. Mellet, ingénieur adjoint subdivisionnaire du contrỏle d'Alger, il résulte que la ligne sur laquelle s'est produit l'accident a été mise en service sans qu'ait été délivrèe l'autorisation préfectorale de circulation de courant prévue par l'article 15 de la loi du 15 juin 1906 ; que c'est là une infraction aux prescriptions édictées dans l'intérêt de la sécurité des personnes dont la pénalité est prévue par l'article 25 de ladite loi ;

Attendu que d'un rapport établi sur l'accident dont s'agit par M. liscande, ingénieur des postes et télégraphes, il résulte que si l'auteur responsable de l'accident a été d'abord le chauffeur dautomobile de la Société S.A.T.A., la Compagnie Lebon a une part de responsabilité parce que le potcau rompu était. insuffisamment robuste, parce qu'elle a commis la faute d'avoir mis en service la ligne dectrique sans avoir pris toutes les précautions que le rapprochement des conducteurs de haute tension al de basse tension, devait entrainer ;

Attendu par suite qu'une des causes de l'accident consiste dans une infraction aux dispositions d'ordre pénal de l'art. 25 de la loi des 15 et 17 juin 1906 et aux dispositions de l'art. 320 , corle pénal ;

Atendu que les tribumax civils sont compétents pour prononcer sur la responsabilite du concessiomaire de travaux publics, résultant des dommages causés anx particuliers, lorsque le fait dommageable alligué, présente le caractìre d'un délit ou d'une contravention, que le clélit ait dé on non poursuivi devant la juridiction repressive ;

Attendu qu'il est dès lors sans intérèt de rechercher si les travaux entrepris par la Compagnie Lebon empruntent le carac- 
tìre de travaux publics à leur objet qui est de procurer à la commune de Saint-Eugène la lumière et la force motrice électrique ;

Attendu qu'il nest pas contesté que la Compagnie Lebon avitit consenti aux époux Casanova une police d'abonnement à l'éclairage électrique (à enregistrer); que le courant clectrique qui a blessé les époux Casanova a été transmis par les fils d'éclairage :

Attendu que le contrat résultant de cette police était par sa nature un contrat de livmaison de foumitures ordinaires de droit privé, n'ayant pas pour objet direet lorganisation ou le fonctionnement du service public articulé par la Compagnie Lehom, que ladite compagnie a manque aux obligations de son contrat en ne prenant pas les précautions nécessaires pour que la livaison de l'éclairage électrique eut lieu, ainsi qu'il était convem, sans danger pour les époux Casamova ;

Attendı que le préjudice pouvant résulter de l'inexéculion de ce contrat est de la compétence des tribunaux de l'ordre judiciaire :

Vu l'art. 130 c. pr. civ.;

Par ces motifs,

Reçoit comme régulier en la forme l'appel interjeté par la Compagnie Lebon du jugement du tribunal civil d'Alger du 21 juin 1925 ;

\section{Confirme ledit jugement ;}

Déboute les parties de leurs autres conchsions;

Condamme l'appelante à l'amende et aux dépens.

Du 30 décembre 1925. - C. d'Alger, $1^{\text {re }}$ ch. - MM. Roche, $1^{\mathrm{er}}$ pr. - Norès, av. gén. - Lefèvre-Paul, Divielle et Soubiranne, av.

Principe général de la compétence du Conseil de Préfecture en matière d'accident. - L'étude d'une exception ne saurait être disjointe d'un aperçu sur le principe : la Juridiction administrative doit être considérée comme compétente, en règle générale, pour statuer sur les conséquences pécuniaires d'un accident causé par les organes de la distribution concédée.

Nous disons et nous précisons "un accident " ce qui signifie la blessure causée à une personne ou à l'animal dont elle est propriétaire, on bien encore la détérioration crée à un objet matéricl qui lui appartient.

On réserve le mot "dommage " au préjudice causé à une propriété immobilière (1).

Et si nous tenons à faire cette distinclion, ce n'est pas pour créer une difficulté, mais au contraire pour en aplanir une. La réparation d'un "dommage "est plus simple que la réparation d'un accident, en ce qu'elle ne comporte aucune exception : la juridiction du Conseil de Préfecture est toujours compétente, et il n'existe pas d'exception, comme en matière d'accidents (2).

(1) Ainsi que le remarque M. Appleton dans sa note D.P. 1923. I. 33 , la loi du 28 pluviôse, an 8 , est muette sur les accidents; elle ne prévoit que les dommages. I,a matière des accidents est en conséquence plutot expliquée par la Jurisprudence que par la loi.

(2) On peut consulter sur ce point une étude que j'ai faite comme Président du Comité du Contentieux de la Chambre Syndicale des Forces Hydrauliques, Communication No 456, annee 1924. Voir notamment le deuxième alinéa cle la page 19 , et y ajouter un arrêt

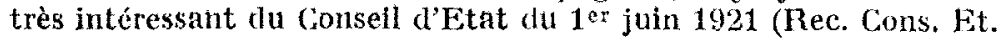

Io principe de la compétence administrative en matière daccident cause par les ouvrages placés et devanl être entretenus par le concessiomaire, so trouve dans l'idée suivante : la plupart des accidents sont occasiomes soit par le vice de l'elablissement de l'ouvage public, soit par une négligence dans le bon entretien de cel ouvrage. Ie concessiomatre tient la place de l'Administration qui, pour des accidents de celte mature, serait incontestablement jugée par le Conseil de Prefecture (1). Il est juste que le Tribunal qui jugerait l' $\Lambda$ dministration pour un fait inhérent à un travail public, soit également compétent à l'encontre de celui qui est le détenteur de ce travail. En tont cas, le principe est certain et accusé par deux décisions aussi intéressantes que récentes.

Lat premiere est une solution du Tribunil des Conllits du 13 janvier 1917 (Rece. Cons. El. page 18 , affaire Guien contre Compagnie generale des Tramwass, voir aussi Communication no 336, année 1919, page 127); un accident a été causé a $11 \mathrm{~m}$ camion qui, conduit par (inieu, est renverse par suile d'un rail qui fait saillie an-dessus du sol : la comnassance du litige appartient au Conscil de Prefecture.

La seconde est un arret de la Cour de Cassation du 27 janvier 1925 (Dalloz Hebdomadaire, amnée 1925, page 127, aff. Sagne contre Chambre de Commerce d'Alger et chemins de fer de Paris-Lyon-Méditeranée). In rail appartenant à la Chambre de Commerce d'Alger et autorisé par un arrèté préfectoral du 21 juillet 1889 faisant saillie au-slessus du sol avait occasionne un accident au sieur Sagne : celui-ci assigna devant le Tribunal Civil, la Chambre de Commerce qui appela en sarantie la Compagnie P.L.M. sur laquelle sle s'etail decharge de l'entretien de la voic. Sur cet appel en garantie, la Cour d'Ager s'étant déclarée incompétente, parce qu'il s'agissait d'un travail public; mais, elle avait statué à l'égard de la Chambre de Commerce et l'avait condamnée à des réparations pécuniaires, en la considérant comme responsable de n'avoir pas exigé de la Compagnie P.L.M. l'entretien que colle-ci avait pris en charge.

1921, page 531, chemins de fer de grande Banlicue contre Préfet de la Marne) : The instance en (lommage sausće il une propriete doit etre intentée par le proprictaire contre la Compagnie concessionnaire quand clle est entreprise apris la remise par l'litat de la ligne à cette Compagnie ; et cette remise, a partir du jour où elle est définitive, libère l'autorité concédante de toute responsabilité pouvant provenir de l'établissement des travaux.

(1) En effet, il ne faut pas perdre de vue que nous étudions ici la situation du concessionnaire : mais, si nous faisions cette étude en ce qui regarde les rapports des tiers et de l'Administration, les solutions seraient encore plus simples. Entre les tiers et l'Administration, le Conseil de Préfecture est toujours compétent. On pourra consulter notre Communication precites, $\mathrm{N}^{\circ}$ 156, anmé 192.4, page 19, 20 alinéa, et les arrets contemus dans la note de la page 22. L'arret le plus probant est celui renclu par le Conseil d'Etat le 16 juillet 1914 (Rec. Cons. Et., année 1914, page 880 et D. 1’. 1920. 3. 5 avec note d'Appleton, voir aussi Communication No 267, année 1915, page 69, alfaire Babouct). Le sieur Babouct avait été blessé au cours d'une opération de pose de fils téléphoniques, faite évidemment par l'Etat et dirigée par un contremaître, le sieur Jajugie, qui avait été conlamné persomnellement pour imprudence dans la direction des travaux par la Juridiction judiclaire. Cette circonstance ne prive pas la vietime du droit de poursuivre l'Etat en réparation pécuniaire de l'aceident. Comme le fait remarquer Appleton dans une note sous les arrêts des 8 et 21 janvier $192: 3$ (D) P. 1923. I. 33, $2^{\circ}$ colome de la nole, in /ine) atcune divergence n'existe entre le Conseil d'Etat sur ce point et la Cour de Cassation; cette derniere admet dans Ies litiges diriges contre l'Administration, la pleine compétence du Conseil de Préfecture (Voir notamment dans la rupture de la bahustrade du Palais de Justice d'Oran l'arrêt clu 2 janvier 1912 (1). P. 1912, page 213, Préfet d'Oran contre Millecam). 
La Cour a cassé sur le chel de pourvoi relatif à la compélence et décidé que l'accident élail dû non pas à un fait d'exploitation, mais au defaut d'entretien d'un ouvrage public; il appartenail donc à la Juridiction administrative de connaîlre du lilige (1).

Seconde exception au principe de la compétence du Conseil de Préfecture. - Nous avons dit ci-dessus que le concessionnaire qui entrelient mal ou a placé d'une façon défeclueuse un ouvrage public, élant considéré comme substitué complètement à l'Adininistration, pouvait réclamer le droit d'être justiciable, en cas d'accident provenant d'un vice de construction ou d'un défaut d'entretien, du 'Tribunal qui aurait jugé ladite $\Lambda$ dministration en raison du mème fail.

Quand il commet une faute d'exploitation, il n'en est plus de même, car il ne s'agit plus de la garde d'un ouvrage public, mais d'un fait essentiellement personnel; rien ne permet de dire que l'Administration aurait commis la même faute. Aussi, (ct c'est la seconde exception que nous ayons à signaler au principe de la compétence du Conseil de Préfecture), le Tribunal de droit commun reprendra tous ses pouvoirs quand il s'agira d'apprécier une faute commise dans l'exploitation. C'est la théorie accusée par la Chambre des Requêtes dans son arrêt du 3 décembre 1918 (affaire Kermina contre Compagnie des Tramways Nogentais) : alle a constaté que laction portée devant le Tribunal de Commerce de Pontoise par Kermina père et fils tendait à obtenir réparation des dégâts causés à une de leurs voitures qui, dans la nuit du 8 août 1914 à St-Mandé, avait heurté un des poteaux supportant la ligne aérienne des Tramways Nogentais et placé sur un refuge que la Compagnie avait été autorisée à construire au milicu de la chaussée. Il n'était pas contesté que le poteau n'étant pas éclairé avait été la cause de l'accident. La Cour décide que ce fait ne se rattache en aucune façon à l'exécution ou à l'entretien d'un service public, et qu'il est susceptible d'être apprécié au point de vue de ses conséquences dommageables, sans qu'il soit nécessaire pour le Juge du fond de s'immiscer dans l'exécution d'un travail public ou de se livrer à l'examen et à l'interprélation du cahier des charges de l'exploitation (voir

(1) M. Appleton (loco cilalo No 231, page 425), fait remarquer l'importance de cet arrèt qui marque une évolution très intéressante de la Cour suprêne, et indique qu'elle se rapproche de la compétence du Conseil d'Etat et du Tribunal des Conflits. le texle de cet arrêt dans la communication $n^{\circ} 336$, année 1919 , page 127).

On peut encore consulter dans le mème sens, un arrêt de la Chambre Civile du 8 janvier 1923 (D.P. 1923. 1. 33, affaire Bouct contre Compagnie générale des Omnibus et la Société des Automobiles le Zèbre); une automobile appartenant à la Société le Zèbre et conduite par Bouet, au service de cette Compagnie, a versé dans une tranchée que faisait pratiquer la Compagnie générale des Omnibus de Paris; la victime alléguait qu'elle était tombée dans un trou que rien ne révélait et aucun ouvrier du chantier n'était présent pour lui en signaler la présence. Ce n'était pas un ouvrage public qui avait causé l'accident, mais une faute d'exploitation. La Cour a rejeté le pourvoi formé contre l'arrêt de la Cour d'Appel de Paris, qui s'était déclarée compétente.

Enfin, dans certains cas, on peut trouver que l'accident provient de deux causes. L'une est inhérente à un établissement défectueux de l'ouvrage public, et l'autre à une absence de précaution. Le type de cette instance est donné par l'arrêt du 24 janvier 1923 (D. P. 1923) I. 35. - Note de M. Appleton affaire de Champlouis contre Compagnie des Tramways du Loiret), l'automobile de De Champlouis avait été tamponnée par un train au passage à niveau de Brinon. Ce passage est dissimulé par des maisons du village et plusieurs accidents s'y sont produits. On reprochait à la Compagnie de ne pas avoir pris les précautions suffisantes pour garantir les passants. La Cour d'Appel d'Orléans s'était déclarée incompétente en déclarant que si l'absence de précautions était certaine, elle ne pouvait pas en apprécier les conséquences indépendamment de la défectuosité de l'établissement du passage qui constitue un travail public. Mais, la Cour de Cassation a décidé que, "l'approbation par "l'autorité compétente des conditions de l'établissement du " passage à niveau, ne dispensait pas la Compagnie des Tram" ways de l'obligation de prendre toute precaution qui aurait " été commandée par la situation des lieux, telle qu'elle avait " été créée par l'acte administratif; qu'il s'agissait là d'un fait "d'exploitation susceptible d'ètre envisagé en lui-mème sans "que les Tribunaux de l'ordre judiciaire aient à s'immiscer dans "l'exécution du travail public, puisque les besoins de la circula" tion sur les routes et les chemins publics qui sont essentielle" ment variables et ne sauraient être prévus avec certitude au " moment de la création des lignes des tramways peuvent, " suivant les circonstances, nécessiter des mesures de précaution " spéciales en dehors de toute faute de construction de la voie "ferrée." 